\title{
Avaliação da qualidade das imagens digitais adquiridas com diferentes resoluções em um sistema de armazenamento de fósforo
}

\section{Evaluation of the quality of digital images acquired with different resolutions in a storage phosphor system}

\author{
Solange Maria de ALMEIDA* \\ Ana Emília Figueiredo de OLIVEIRA** \\ Gisela André PAGANINI** \\ Francisco HAITER NETO* \\ Frab Norberto BÓSCOLO***
}

\begin{abstract}
ALMEIDA, S. M.; OLIVEIRA, A. E. F.; PAGANINI, G. A.; HAITER NETO, F.; BÓSCOLO, F. N. Avaliação da qualidade das imagens digitais adquiridas com diferentes resoluções em um sistema de armazenamento de fósforo. Pesqui Odontol Bras, v. 14, n. 3, p. 262-267, jul./set. 2000.
\end{abstract}

\begin{abstract}
O objetivo do presente trabalho foi avaliar comparativamente a qualidade das imagens adquiridas, por meio da placa de fósforo, com três diferentes resoluções - 150, 300 e 600 dpi, e a eficiência de alguns recursos de manipulação disponíveis no software do sistema estudado. Os objetos de análise constituíram-se de quinze dentes com a lima 8 em seu interior, cujas mensurações endodônticas foram realizadas por cinco avaliadores. O sistema digital utilizado foi o DenOptix (Dentsply International/Gendex Dental X-Ray Division, Des Plaines, IL). Os resultados mostraram diferença estatística entre as diferentes resoluções empregadas, com as imagens adquiridas com 150 dpi apresentando-se de qualidade inferior às de 300 e 600 dpi, estas equivalentes. Os diferentes recursos digitais empregados não se apresentaram estatisticamente significantes entre si. Com base nos resultados, conclui-se que as imagens de 150 dpi apresentam-se com qualidade inferior àquelas adquiridas com 300 e 600 dpi, que oferecem similar nível de eficiência, imagens em condições satisfatórias para uma análise radiográfica. Quanto a aplicação dos diferentes recursos de manipulação estudados, pode-se concluir, em função dos resultados equivalentes que apresentaram, que, em mensurações endodônticas, a escolha de se trabalhar com um recurso isoladamente ou em associação fica ao encargo de um critério subjetivo de seleção.
\end{abstract}

UNITERMOS: Radiografia dentária; Intensificação de imagem radiográfica; Endodontia; Odontometria.

\section{INTRODUÇÃO}

O objetivo principal da realização de qualquer tipo de tomada radiográfica é a reprodução mais fiel possivel do objeto em interesse, pois a qualidade de imagem se traduz no elemento de fundamental importância para o direcionamento de um radiodiagnóstico. Aliado a isto, depara-se muitas vezes com outras questões que devem ser consideradas no momento da aquisição de uma radiografia digital, e dentre várias, cita-se o tempo de trabalho e o tamanho de seu arquivo.

Abordando-se essas questões, desenvolveu-se este trabalho com um novo sistema digital de armazenamento de fósforo, o DenOptix (Dentsply
International/Gendex Dental X-Ray Division, Des Plaines, IL). Este sistema possui um recurso que possibilita que as radiografias intrabucais sejam adquiridas com diferentes resoluções, no caso, 150,300 e 600 dpi, levando conseqüentemente a uma variação no tamanho do pixel, na resolução espacial da imagem $(\mathrm{pl} / \mathrm{mm})$, assim como no tamanho da sua matriz. Destaca-se aqui que estes fatores, de relevante importância na qualidade de uma radiografia digital ${ }^{1,2,4,5,7,9,10,13,14}$, comportam-se como conceitos intimamente relacionados, com influência direta ainda no tempo de trabalho, na magnificação da imagem, assim como no tamanho do seu arquivo.

\footnotetext{
* Professores Assistentes Doutores; ** Doutorandas; *** Professor Titular - Disciplina de Radiologia da Faculdade de Odontologia de Piracicaba da UNICAMP.
} 
ALMEIDA, S. M.; OLIVEIRA, A. E. F.; PAGANINI, G. A.; HAITER NETO, F.; BÓSCOLO, F. N. Avaliação da qualidade das imagens digitais adquiridas com diferentes resoluções em um sistema de armazenamento de fósforo. Pesqui Odontol Bras, v. 14, n. 3, p. 262-267, jul./set. 2000.

Portanto, em virtude de este sistema oferecer condições de se trabalhar com diferentes resoluções, resolveu-se avaliar comparativamente a qualidade das imagens adquiridas com 150, 300 e 600 dpi, por meio de mensurações endodônticas, verificando-se ainda se a "manipulação do brilho e contraste" e a aplicação dos recursos "3D" e "negativo" aumentam a fidelidade das medidas efetuadas.

\section{MATERIAIS E MÉTODOS}

O sistema digital estudado na presente pesquisa foi o sistema de armazenamento de fósforo, DenOptix (Dentsply International/Gendex Dental X-Ray Division, Des Plaines, IL), que utilizou os seguintes componentes: DenOptix laser "scanner", carrossel do "scanner" (Ceph back) para o posicionamento das placas ópticas, placas de fósforo tamanho $31 \times 41 \mathrm{~mm}$, software inerente ao sistema e envoltórios plásticos protetores da placa. Acoplado ao conjunto do sistema, foi utilizado um computador Pentium $200 \mathrm{MHz}, 64$ memória RAM, acoplado a um monitor S-VGA, tela plana, 17 polegadas, configuração de tela de 1.024 x 768 pixels de resolução e placa de vídeo de $2 \mathrm{MB}$. As características gerais do sensor utilizado encontram-se na Tabela 1 .

Os objetos de estudo constituíram-se em 15 dentes unirradiculares, montados em troquéis individuais confeccionados em gesso com pó de serragem simulando tecido ósseo, com lima endodôntica $\mathrm{n}^{\circ} 8$ (Maillefer Instruments CH 1338, Ballaigues, Swiss) no seu interior. Cada dente possuía um número de identificação próprio.

A escolha por dentes unirradiculares deu-se em função de estes apresentarem uma maior facilidade para o posicionamento da lima, evitando-se assim a tensão que os dentes multirradiculares tendem a fazer no instrumento endodôntico, o que poderia levar a uma grande variabilidade de resultados interexaminadores, em virtude da escolha de pontos diferentes de marcação no momento da re- alização das mensurações digitais. Os dentes unirradiculares tiveram suas bordas incisais aplainadas, definindo-se, assim, melhor os pontos superiores a serem marcados.

$\mathrm{O}$ aparelho de raios $\mathrm{X}$ utilizado para aquisição das radiografias, foi o GE 1000 (General Electric Company, Milwaukee, WI, USA), operando com $65 \mathrm{kVp}$, corrente de $10 \mathrm{~mA}$, distância foco-objeto de $32 \mathrm{~cm}$ e tempo de exposição de $0,2 \mathrm{~s}$, tempo, este, selecionado em um teste piloto, no qual se procurou definir o menor tempo capaz de fornecer uma imagem ideal.

Para as tomadas, medições de dose foram realizadas, o que permitiu uma avaliação mais objetiva da relação de tempo de exposição versus dose. Para isto, utilizou-se a câmara de ionização Victoreen 06-526, o "timer" de RX Victoreen 07-457 (Victoreen Inc., Cleveland, Ohio) e o " $\mathrm{kVp}$ meter" Unfors Inst. - 9002 (Unfors Instruments, Billdal, Sweden). Os resultados mostraram que o aparelho utilizado apresentou exatidão na reprodutibilidade das respostas, no que concerne ao tempo de exposição, à quilovoltagem e à dose.

Os troquéis foram posicionados sobre uma bancada de trabalho de forma padronizada, com um número de identificação de chumbo. Em cada dente foi introduzida uma lima endodôntica $n^{\circ} 8$, cujo comprimento de trabalho foi preestabelecido em um gabarito próprio que continha as medidas reais de trabalho, confeccionado aleatoriamente sem que tivesse relação com o comprimento do dente, a fim de que os examinadores não fossem induzidos no ato da marcação. A placa de fósforo foi posicionada paralelamente ao troquel e o cilindro direcionado de forma que o feixe central incidisse perpendicularmente ao objeto e à placa, com ângulo vertical e horizontal de $0^{\circ}$. Cada troquel foi radiografado três vezes, pois três foram as leituras efetuadas pelo "scanner": 150, 300 e 600 dpi. A escolha por tomadas individuais para cada tipo de leitura ocorreu em virtude da degradação observada na imagem fornecida pela placa cuja leitura já ha-

TABELA 1 - Características gerais da placa de fósforo do sistema DenOptix, de acordo com a forma de leitura realizada pelo "scanner".

\begin{tabular}{|c|c|c|c|c|c|c|}
\hline & \multirow{2}{*}{$\begin{array}{l}\text { Matriz encontrada } \\
\quad \text { (pixels) }\end{array}$} & \multirow{2}{*}{$\begin{array}{l}\text { Tamanho do } \\
\text { pixel }^{2}\end{array}$} & \multirow{2}{*}{$\begin{array}{l}\text { Resolução } \\
\text { espacial }^{2}\end{array}$} & \multirow{2}{*}{$\begin{array}{l}\text { Arquivo de imagem } \\
\text { (TIFF) }\end{array}$} & \multicolumn{2}{|c|}{ Tempo de leitura do "scanner" } \\
\hline & & & & & 1 placa & 8 placas \\
\hline $150 \mathrm{dpi}$ & $184 \times 243$ & $170 \mu \mathrm{m}$ & $4 \mathrm{pl} / \mathrm{mm}$ & $45 \mathrm{kB}$ & $0: 36 \mathrm{~s}$ & $1: 54 \mathrm{~s}$ \\
\hline 300 dpi & $367 \times 485$ & $85 \mu \mathrm{m}$ & $6 \mathrm{pl} / \mathrm{mm}$ & $175 \mathrm{kB}$ & $1: 12 \mathrm{~s}$ & $3: 49 \mathrm{~s}$ \\
\hline $600 \mathrm{dpi}$ & $969 \times 733$ & $42 \mu \mathrm{m}$ & $9 \mathrm{pl} / \mathrm{mm}$ & $695 \mathrm{kB}$ & $2: 25 \mathrm{~s}$ & $7: 39 \mathrm{~s}$ \\
\hline
\end{tabular}


ALMEIDA, S. M.; OLIVEIRA, A. E. F.; PAGANINI, G. A.; HAITER NETO, F.; BÓSCOLO, F. N. Avaliação da qualidade das imagens digitais adquiridas com diferentes resoluções em um sistema de armazenamento de fósforo. Pesqui Odontol Bras, v. 14, n. 3, p. 262-267, jul./set. 2000 .

via sido realizada, pois este sistema não apaga a energia residual da placa após exibição da imagem.

Participaram como examinadores cinco radiologistas dos quais um era também endodontista. Foi realizada uma demonstração prática, individual, com cada examinador, ensinando-os como trabalhar com o software do DenOptix e visando, ainda, a padronização de seus trabalhos. Dessa forma, fez-se então, a calibragem dos examinadores deixando-os aptos a iniciarem as mensurações.

Na metodologia de análise, constava a manipulação do "brilho e do contraste", "3D" e "negativo", já que um dos propósitos do trabalho foi verificar a eficiência individual de cada um destes recursos nas mensurações endodônticas. Portanto, cada examinador realizou 135 mensurações, pois 15 eram os objetos, 3 os tipos de processamento digital da imagem e 3 as resoluções. Destaca-se aqui que foi aconselhado, a cada um dos examinadores, a limitação do número de mensurações/dia, prevenindo-se um possivel cansaço visual que poderia interferir no momento da marcação dos pontos.

Foi permitida ainda ao examinador a utilização dos recursos "equalizar" e "zoom", que poderiam ser utilizados em conjunto com os outros três recursos obrigatoriamente empregados. O primeiro, pela preferência de alguns em trabalhar com radiografias mais densas que o padrão; e o segundo, em função do tamanho com que as imagens de 150 dpi se apresentavam na tela do monitor.

Cada examinador registrou as medidas em tabelas confeccionadas para este fim, sendo estes dados submetidos à análise estatística.

\section{RESULTADOS}

Foram obtidas as diferenças entre medidas endodônticas feitas por cinco avaliadores e os respectivos gabaritos em 15 imagens radiográficas, segundo a resolução e o recurso utilizado. Essas diferenças foram submetidas à análise de variância, em esquema fatorial. Os resultados estão apresentados na Tabela 2 . Os valores de $F$ indicam que existe diferença estatística ao nível de 5\% de significância, entre as médias das diferenças das medidas endodônticas e os respectivos gabaritos feitas com diferentes resoluções, e entre as médias das diferenças das medidas endodônticas e os respectivos gabaritos feitas por diferentes avaliadores.

Observa-se ainda, com base na Tabela 2, que as diferenças dos resultados dos diferentes recursos digitais utilizados não se apresentaram estatisticamente significantes entre si.

As médias das diferenças entre as medidas e os respectivos gabaritos para cada resolução, em ordem decrescente, estão apresentados na Tabela 3. Essas médias foram comparadas pelo teste de Tukey. Como as médias referem-se à diferença entre a medida feita pelo avaliador e o respectivo gabarito, a resolução é tanto melhor quanto mais próximo de zero estiver a média.

Considerando-se os resultados do teste de Tukey contidos na Tabela 3, pode-se concluir que as imagens de 300 e 600 dpi têm registros mais fiéis que as de $150 \mathrm{dpi}$.

É interessante notar na Tabela 2 que existe diferença estatística entre os avaliadores, que se refere à diferença entre a medida e o respectivo gabarito, porém não foi feita uma comparação das

TABELA 2 - Análise de variância das diferenças entre medidas endodônticas e os respectivos gabaritos.

\begin{tabular}{l|r|r|r|r|r}
\hline \hline \multicolumn{1}{c|}{ Causas } & $\begin{array}{r}\text { Graus de } \\
\text { liberdade }\end{array}$ & $\begin{array}{c}\text { Soma } \\
\text { dos qua- } \\
\text { drados }\end{array}$ & $\begin{array}{r}\text { Qua- } \\
\text { drado } \\
\text { médio }\end{array}$ & $F$ & p-valor \\
\hline Recurso & 2 & 0,58 & 0,29 & 0,38 & 0,6869 \\
\hline Resolução & 2 & 40,73 & 20,37 & 26,40 & 0,0000 \\
\hline Radiografia & 14 & 87,74 & & & \\
\hline Avaliador & 4 & 12,01 & 3,00 & 3,89 & 0,0039 \\
\hline $\begin{array}{l}\text { Recurso } \\
\text { versus } \\
\text { resolução }\end{array}$ & 4 & 1,81 & 0,45 & 0,59 & 0,6722 \\
\hline $\begin{array}{l}\text { Recurso } \\
\text { versus } \\
\text { avaliador }\end{array}$ & 8 & 10,08 & 1,26 & 1,63 & 0,1119 \\
\hline $\begin{array}{l}\text { Resolução } \\
\text { versus } \\
\text { avaliador }\end{array}$ & 8 & 4,68 & 0,58 & 0,76 & 0,6404 \\
\hline Erro & 632 & 487,52 & 0,77 & & \\
\hline Total & 674 & 645,16 & & & \\
\hline \hline
\end{tabular}

TABELA 3 - Médias das diferenças entre as medidas e o respectivo gabarito para cada resolução.

\begin{tabular}{c|c|c}
\hline \hline Resolução & Média & Teste \\
\hline 150 & 0,539 & $\mathrm{~A}$ \\
\hline 300 & 0,059 & $\mathrm{~B}$ \\
\hline 600 & 0,015 & $\mathrm{~B}$ \\
\hline \hline
\end{tabular}

Nota: letras diferentes indicam diferença estatística. 
ALMEIDA, S. M.; OLIVEIRA, A. E. F.; PAGANINI, G. A.; HAITER NETO, F.; BÓsCOLO, F. N. Avaliação da qualidade das imagens digitais adquiridas com diferentes resoluções em um sistema de armazenamento de fósforo. Pesqui Odontol Bras, v. 14, n. 3 , p. 262-267, jul./set. 2000.

médias de avaliadores pelo teste de Tukey, por não existir interesse em se identificar o(s) melhor(es) avaliador(es).

\section{DISCUSSÃO}

Dentro da Radiologia, encontram-se, com freqüência, registros radiográficos diminutos, cuja detecção faz-se fundamental para um diagnóstico precoce, como por exemplo, cáries incipientes. No caso da Endodontia, o profissional freqüentemente depara-se com canais atrésicos, que exigem que o trabalho inicial seja realizado com limas de registros radiográficos sutis. Portanto, selecionou-se a lima 8 como instrumento de trabalho, por exigir para toda sua evidenciação, uma radiografia de excelente padrão, o que se fez importante para uma análise comparativa da qualidade de imagem. A seleção deste instrumento endodôntico propiciou ainda mais objetividade dentro da proposta de trabalho, em função do conhecimento do comprimento real do objeto de análise em cada uma das tomadas realizadas.

Os resultados apontaram uma equivalência nas mensurações realizadas nas imagens adquiridas com 300 e $600 \mathrm{dpi}$, em detrimento àquelas de $150 \mathrm{dpi}$, que apresentaram resposta inferior. Logo, se as imagens de 300 e de 600 dpi não apresentaram diferença estatística nas mensurações com a lima 8, dificilmente estes resultados apresentariam respostas diferentes em relação a qualquer outro objeto na prática clínica, pois raramente o profissional depara-se com registros tão diminutos quanto o extremo do instrumento em questão. Dos resultados inferiores apresentados pelas imagens adquiridas com 150 dpi, deduz-se que é inviável a utilização desta resolução para o diagnóstico de registros radiográficos sutis.

O tamanho do pixel está relacionado à resolução espacial da imagem em $\mathrm{pl} / \mathrm{mm}^{2,5,7,9,13,14}$, e quanto menor o tamanho do pixel maior a resolução. Portanto, as imagens de 150 dpi possuem um tamanho de pixel maior e conseqüentemente uma resolução espacial menor, de aproximadamente $4 \mathrm{pl} / \mathrm{mm}$, enquanto as de $300 \mathrm{dpi}$ apresentam em torno de $6 \mathrm{pl} / \mathrm{mm}$ e as de $600 \mathrm{dpi}$ por volta de $9 \mathrm{pl} / \mathrm{mm}$. O que se pode observar com estes resultados é que a grande diferença em termos de diagnóstico situam-se entre a resolução espacial de $4 \mathrm{e}$ $6 \mathrm{pl} / \mathrm{mm}$, pois de 6 para $9 \mathrm{pl} / \mathrm{mm}$ não se observou diferença estatisticamente significante. Isto está de acordo com a citação de alguns autores, que ressaltam a boa qualidade de imagem da placa de fósforo de resolução espacial de $6 \mathrm{pl} / \mathrm{mm}$ nas tarefas de diagnóstico ${ }^{1,2,4,5,7,9}$.

Um fator importante a ser considerado, na seleção da resolução da imagem em dpi, é o tempo de trabalho gasto na aquisição da radiografia. Logo, é preferivel utilizar-se a resolução de 300 dpi, que é o padrão do sistema para radiografias intra-orais, que a de $600 \mathrm{dpi}$, pois com isto reduz-se pela metade o tempo de trabalho do "scanner" para realização da leitura da placa. Além disso, pode-se considerar que as imagens de 600 dpi demoram mais para serem arquivadas, abertas, importadas ou exportadas, além de consumirem uma acentuada memória de arquivo. Destaca-se que os tempos de leitura do DenOptix "scanner" para 300 e 600 dpi (Tabela 1) são altos se considerarmos a média do tempo de aquisição das radiografias digitais. Porém, este tempo de trabalho se tornará mais rentável, com o aumento de placas posicionadas no carrossel. Vale ressaltar que deverá ser adicionado ao tempo de trabalho o tempo gasto na dessensibilização da placa após a leitura, pois o referido sistema não a oferece pronta para o uso após o fornecimento da imagem na tela do monitor, pelo fato de o DenOptix "scanner" não eliminar a energia residual da placa após a realização de sua leitura.

Apesar de as imagens de $150 \mathrm{dpi}$, serem as de mais rápida aquisição, pode-se ter um gasto maior no tempo de trabalho, se for necessária a repetição da tomada, dada a dificuldade em se interpretar alguma região de interesse. Além disso, não se recomenda para um diagnóstico inicial a utilização de 150 dpi, pois algum registro radiográfico sutil, mas de importância para o diagnóstico, poderá passar despercebido. Portanto destaca-se que a resolução de 150 dpi só deverá ser utilizada, se o objeto de análise não exigir muita qualidade da radiografia, e somente nos casos em que o tempo de trabalho e o arquivo da imagem se constituírem em fatores relevantes.

Um ponto importante a ser considerado e de influência direta nos resultados encontrados é o fator magnificação da imagem. Tal condição está relacionada ao tamanho da matriz da imagem e, portanto, as de $150 \mathrm{dpi}$, por possuírem uma matriz de apenas 184 x 243 pixels, apresentam-se na tela do monitor de tamanho bem mais reduzido do que a média das radiografias digitais, o que leva a uma necessidade da utilização do recurso "zoom". Porém, tal recurso é um auxiliar limitado, pois com seu aumento progressivo, acentua-se também a evidenciação dos pixels, o que dificulta o diagnóstico. VERSTEEG et al. ${ }^{12}$ (1998) citam que a redução do tamanho da imagem digital deve levar a um 
ALMEIDA, S. M.; OLIVEIRA, A. E. F.; PAGANINI, G. A.; HAITER NETO, F.; BÓSCOLO, F. N. Avaliação da qualidade das imagens digitais adquiridas com diferentes resoluções em um sistema de armazenamento de fósforo. Pesqui Odontol Bras, v. 14, n. 3, p. 262-267, jul./set. 2000 .

comprometimento do diagnóstico, o que coincide com os resultados de SVANAES et al. ${ }^{9}$ (1996), em que as imagens analisadas sem magnificação no diagnóstico de cárie, apresentaram resultados significantemente inferior àquelas analisadas com magnificação. Isto foi comprovado aqui com as imagens de 150 dpi, e não teve correspondência com as de $300 \mathrm{dpi}$, pelo fato de estas já se apresentarem em tamanho satisfatório na tela do monitor, tendo-se nos resultados um indicativo disto. Porém, se o profissional achar necessário acionar o "zoom", este não requisitará tanto aumento, como no caso das radiografias de $150 \mathrm{dpi}$. Com as imagens de $600 \mathrm{dpi}$, tem-se muitas vezes que utilizar a barra de rolagem, pois a radiografia apresenta-se maior que a tela do monitor, o que nem sempre torna-se prático, quando se quer analisar a radiografia por inteiro. MOYSTAD et al. ${ }^{6}(1995)$ ao estudarem cinco diferentes magnificações, X 3, X 6, X 12, X 18, X 30, encontraram resultados significantemente inferior nas duas mais altas e similares nas três primeiras, o que representa que uma magnificação acentuada pode prejudicar a análise radiográfica, assim como diferentes magnificações localizadas dentro de uma certa amplitude podem não vir a interferir no resultado final de um radiodiagnóstico.

Quanto a eficiência dos recursos de manipulação aplicados para a realização das mensurações, não se observou diferença estatisticamente significante entre eles. Portanto, a escolha de qual recurso trabalhar fica ao encargo de um critério subjetivo de preferência.

Alguns trabalhos ${ }^{8,11}$ relatam limitação da placa de fósforo nas mensurações de lima 10, resultado que diverge dos encontrados neste estudo, pois o sistema DenOptix forneceu uma qualidade de imagem satisfatória, o que pode ser observado por meio dos valores reduzidos das médias das diferenças entre as medidas realizadas e o gabarito das medidas reais, nas resoluções de 300 e 600 dpi (Tabela 3). A eficiência da placa de fósforo em detectar o baixo ${ }^{3,4,7}$ constitui-se num fator de fundamental importância para o satisfatório registro da lima endodôntica.

Observando-se os resultados, nota-se que existe uma diferença estatística entre as mensurações realizadas pelos examinadores. Contudo, essa condição corresponde a um aspecto subjetivo de análise relacionado à acuidade visual de cada um e a eliminação ou a substituição de algum examinador eliminaria esta variabilidade clínica comumente encontrada.

\section{CONCLUSÕES}

Com base nos resultados encontrados, pode-se concluir que as imagens de 150 dpi se apresentam com qualidade inferior àquelas adquiridas com 300 e $600 \mathrm{dpi}$, que oferecem, com similar nivel de eficiência, condições satisfatórias para uma análise radiográfica. Quanto à aplicação dos diferentes recursos de manipulação estudados, pôde-se concluir, em função dos resultados equivalentes que apresentaram, que, em mensurações endodônticas, a escolha de se trabalhar com um recurso isoladamente ou em associação fica ao encargo de um critério subjetivo de seleção, que tende a se tornar mais eficiente de acordo com a familiaridade adquirida no decorrer da prática clínica.

\footnotetext{
ALMEIDA, S. M.; OLIVEIRA, A. E. F.; PAGANINI, G. A.; HAITER NETO, F.; BÓSCOLO, F. N. Evaluation of the quality of digital images acquired with different resolutions in a storage phosphor system. Pesqui Odontol Bras, v. 14, n. 3, p. 262-267, jul./set. 2000 .
}

\begin{abstract}
The aim of this paper was to evaluate the quality of the image acquired with the DenOptix storage phosphor plate (Dentsply International/Gendex Dental X-Ray Division, Des Plaines, IL), with three different resolutions - 150, 300 and $600 \mathrm{dpi}$, as well as the efficiency of some tools that are available in such a kind of software. The objects analyzed were 15 teeth containing a \#8 endodontic file in their root canal. Endodontic measurements were performed by 5 examiners. The results showed a statistically significant difference between the employed resolutions. The $150 \mathrm{dpi}$ images had inferior quality when compared with the 300 and $600 \mathrm{dpi}$ images, which were similar to each other. The different digital tools used did not show statistically significant difference. According to the results it was concluded that the images obtained with 150 dpi were worse than those obtained with 300 and $600 \mathrm{dpi}$, which presented similar efficiency and good image quality for radiographic analysis. Based on the similar results obtained with the different digital tools analyzed, it was concluded that, in endodontic measurements, the choice between working with only one tool or with associations of two or more tools, depends upon a subjective criterion of selection.
\end{abstract}

UNITERMS: Radiography, dental; Radiographic image enhancement; Endodontics; Odontometry. 
ALMEIDA, S. M.; OLIVEIRA, A. E. F.; PAGANINI, G. A.; HAITER NETO, F.; BÓSCOLO, F. N. Avaliação da qualidade das imagens digitais adquiridas com diferentes resoluções em um sistema de armazenamento de fósforo. Pesqui Odontol Bras, v. 14, n. 3, p. 262-267, jul./set. 2000.

\section{REFERÊNCIAS BIBLIOGRÁFICAS}

1. CONOVER, G. L.; HILDEBOLT, C. F.; YOKOYAMACROTHERS, N. Comparison of linear measurements made from storage phosphor and dental radiographs. Dentomaxillofac Radiol, v. 25, n. 5, p. 268-273, Nov. 1996.

2. GRÖNDAHL, H. G.; WENZEL, A.; BORG, E.; TAMMISALO, E. An image plate system for digital intra-oral radiography. Dent Update, v. 23, n. 8, p. 334-337, Oct. 1996.

3. HILDEBOLT, C. F.; FLETCHER G.; YOKOYAMACROTHERS, N.; CONOVER, G. L.; VANNIER, M. W. A comparison of the response of storage phosphor and film radiography to small variations in X-ray exposure. Dentomaxillofac Radiol, v. 26, n. 3, p. 147-151, May 1997.

4. HUDA, W.; RILL, L. N.; BENN, D. K.; PETTIGREW, J. C. Comparison of a photostimulable phosphor system with film for dental radiology. Oral Surg Oral Med Oral Pathol Oral Radiol Endod, v. 83, n. 6, p. 725-731, June 1997.

5. LUOSTARINEN, T.; TAMMISALO, T.; VÄHÄTALO, K.; TAMMISALO, E. Comparison of intra-oral digital and film radiography for diagnosis of periapical bone lesions. Dentomaxillofac Radiol, v. 24, n. 2, p. 92-93, May 1995

6. MOYSTAD, A.; SVANAES, D. B.; LARHEIM, T. A.; GRÖNDAL, H. G. Effect of image magnification of digitized bitewing radiographs on approximal caries detection: an in vitro study. Dentomaxillofac Radiol, v. 24, n. 4, p. 255-259, Nov. 1995.

7. OLIVEIRA, A. E. F. Avaliação da qualidade de imagem, sensibilidade e escala dinâmica de três sistemas digitais (Sens-A-Ray, Digora, CDR), filme E-speed e filme digitalizado. Piracicaba, 1999. 115 p. Dissertação (Mestrado) - Universidade Estadual de Campinas.

8. SCHMAGE, P.; NERGITZ, I.; PFEIFFER, P.; PLATZER, U. Diagnostic accuracy of in vitro digital radiographs. Dentomaxillofac Radiol, v. 26, p. 274-275, Sept. 1997.

9. SVANAES, D. B.; MOYSTAD, A.; RISNES, S.; LARHEIM, T. A.; GRÖNDAL, H. G. Intraoral storage phosphor radiography for approximal caries detection and effect of image magnification: comparison with conventional radiography. Oral Surg Oral Med Oral Pathol Oral Radiol Endod, v. 82, n. 1, p. 94-100, July 1996.

10. VANDRE, R. H.; WEBBER, R. L. Future trends in dental radiology. Oral Surg Oral Med Oral Pathol Oral Radiol Endod, v. 80, n. 4, p. 471-478, Oct. 1995.

11. VELDERS, X. L.; SANDERINK, G. C.; VAN DER STELT, P. F. Dose reduction of two digital sensor systems measuring file lenghts. Oral Surg Oral Med Oral Pathol Oral Radiol Endod, v. 81, n. 5, p. 607-612, May 1996.

12. VERSTEEG, C. H.; SANDERINK, G. C.; LOBACH, S. R.; VAN DER STELT, P. F. Reduction in size of digital images: does it lead to less detectability or loss of diagnostic information? Dentomaxillofac Radiol, v. 27, n. 2, p. 93-96, Mar. 1998.

13. WENZEL, A. Computer-aided image manipulation of intraoral radiographs to enhance diagnosis in dental practice: a review. Int Dent J, v. 43, n. 2, p. 99-108, Apr. 1993.

14. WENZEL, A.; GRÖNDAHL, H. G. Direct digital radiography in the dental office. Int Dent J, v. 45, n. 1, p. 27-34, Feb. 1995.
Recebido para publicação em 16/09/99 Enviado para reformulação em 05/04/00 Aceito para publicação em 26/06/00 